PACS 32.15Rm;

УДК 539.184

\title{
SENSING THE HYPERFINE STRUCTURE AND NUCLEAR QUADRUPOLE MOMENT FOR RADIUM
}

\author{
E. P. Gurnitskaya, O. Yu. Khetselius \\ Odessa National Polytechnical University, Odessa
}

\begin{abstract}
SENSING THE HYPERFINE STRUCTURE AND NUCLEAR QUADRUPOLE MOMENT FOR RADIUM

E. P. Gurnitskaya, O. Yu. Khetselius

It has been carried out sensing and estimating the hyperfine structure parameters and nuclear quadrupole moment of the radium on the basis of gauge-invariant QED perturbation theory with an account of correlation effects
\end{abstract}

Key words: sensing and estimate, hyperfine structure, nuclear quadrupole moment, radium

\section{Анотація \\ ДЕТЕКТУВАННЯ СУПЕРТОНКОЇ СТРУКТУРИ І ВИЗНАЧЕННЯ ЯДЕРНОГО КВАДРУПОЛЬНОГО МОМЕНТУ ДЛЯ РАДІЯ}

О. П. Гурницька, О. Ю. Хещеліус

Виконано детектування та оцінка параметрів супертонкої структури та ядерного квадрупольного моменту для радія на підставі калібровочно-інваріантної КЕД теорії збурень 3 урахуванням кореляційних ефектів.

Ключові слова: детектування та оцінка, супертонка структура, ядерний квадрупольний момент, радій

\section{Аннотация}

\section{ДЕТЕКТРОВАНИЕ СВЕРХТОНКОЙ СТРУКТУРЫ И ОПРЕДЕЛЕНИЕ ЯДЕРНОГО КВАДРУПОЛЬНОГО МОМЕНТА ДЛЯ РАДИЯ}

\section{Е. П. Гурницкая, О. Ю. Хецелис}

Выполнено детектирование и оценка параметров сверхтонкой структуры и ядерного квадрупольного момента для радия на основе калибровочно-инвариантной КЭД теории возмущений с учетом корреляционных эффектов.

Ключевые слова: детектирование и оценка, сверхтонкая структура, ядерный квадрупольный момент, радий 
In last years a sensing the hyperfine structure parameters and nuclear quadrupole moments for different heavy elements attracts a great interest (c.f.[1-15]). It is provided by necessity of further developing the modern as atomic and as nuclear theories. From the other side, a great progress in experiments has been achieved [1-4]. In calculations of the heavy ions the well known multi-configuration (MC) Dirac-Fock (DF) approach is widely used [1]. It provides the most reliable version of calculation for atomic systems. More effective method, based on the quantum electrodynamics (QED) perturbation theory (PT), has been developed in the series of papers [7-14, 17-20]. The further improvement of this method is connected with using the gauge invariant procedures of generating relativistic orbitals basis's and more correct treating the nuclear and quantum electrodynamics effects.

In references [7-9] it has been developed a new $a b$ initio approach to calculating spectra of heavy systems with account of relativistic, correlation effects, based on the QED qauge-invariant perturbation theory and new effective procedures for accounting the nuclear and radiative corrections in the hyperfine structure calculation. Here we carry out sensing and estimating the hyperfine structure parameters and nuclear quadrupole moment of the radium on the basis of gauge-invariant QED PT with an account of relativistic, correlation, nuclear finite size and radiative effects. Let us describe in brief the important moments of the calculation procedure.. Full details of the whole method and corresponding numerical procedure of calculation of the different characteristics, including the hyperfine structure constants and nuclear quadrupole moment, can be found in [5-14].

The wave functions zeroth basis is found from the Dirac equation solution with potential, which includes the core ab initio potential, electric, polarization potentials of nucleus (the gaussian form for charge distribution in the nucleus is used). All correlation corrections of the second and high orders of perturbation theory (electrons screening, particle-hole interaction etc.) are accounted for $[6,7]$. We set the charge distribution in the nucleus by the Gaussian function:

$$
\begin{gathered}
\rho(r \mid R)=\left(4 \gamma^{3 / 2} / \sqrt{\pi}\right) \exp \left(-\gamma r^{2}\right) \\
\int_{0}^{\infty} d r r^{2} \rho(r \mid R)=1 ; \int_{0}^{\infty} d r r^{3} \rho(r \mid R)=R
\end{gathered}
$$

Here $\gamma=4 / \pi R^{2} ; R$ is an effective nucleus radius, defined as:

$$
R=1.60 \times 10^{-13} z^{1 / 3}(\mathrm{~cm}) .
$$

Such definition of an effective nuclear radius is to be suitable at least as some zeroth approximation. Our approach allows to calculate the derivatives on $\mathrm{R}$ for characteristics which describe interaction of a nucleus with the external electrons. Then it is possible to make the redistribution of results when a radius $\mathrm{R}$ is varied within the physically reasonable limits. As it has been shown in many papers (e.g. papers $[1-5,7,11]$ and refs there), the models with the Fermi and Gauss charge distribution in a nucleus are most widespread and more correct in comparison with the model of homogeneous ball charge distribution. For example, let us mention that a difference in values of the spectra levels energies is about several $\mathrm{cm}^{-1}[4,5,7]$. At the same time the most advanced model must be based on the direct solving of the corresponding nuclear task.. As example, one could mention different versions of the shell model with the Woods-Saxon and spin-orbit potentials (e.g. refs.[16-20]). As a preliminary comprehensive analysis shows, using these models could increase an accuracy of definition of the hyperfine structure constants on several units (decades) $\mathrm{MHz}$. From other side, using the smooth function of the charge distribution insist of the non-smoth one is more effective as it simplifies the calculational procedure and allows to make more realistic modelling the charge distribution. Let us suppose that the point-like nucleus posseses by some central potential $W(R)$. The transition to potential of the finite nucleus is realized by substituition $W(r)$ on

$$
\begin{gathered}
W(r \mid R)=W(r) \int_{0}^{r} d r r^{2} \rho(r \mid R)+ \\
+\int_{r}^{\infty} d r \quad r^{2} W(r) \rho(r \mid R)
\end{gathered} .
$$

In our case the Coulomb potential for spherically symmetric density $\rho(r \mid R)$ is:

$$
V_{\text {nucl }}(r \mid R)=-\left((1 / r) \int_{0}^{r} d r^{\prime} r^{\prime 2} \rho\left(r^{\prime} \mid R\right)+\int_{r}^{\infty} d r^{\prime} r^{\prime} \rho\left(r^{\prime} \mid R\right)\right.
$$

This potential is calculated from solving the following system of differential equations: 


$$
\begin{gathered}
V^{\prime} \text { nucl }(r, R)=\left(1 / r^{2}\right) \int_{0}^{r} d r^{\prime} r^{\prime 2} \rho\left(r^{\prime}, R\right) \equiv\left(1 / r^{2}\right) y(r, R) \\
y^{\prime}(r, R)=r^{2} \rho(r, R) \\
\rho^{\prime}(r, R)=-8 \gamma^{5 / 2} r / \sqrt{\pi} \exp \left(-\gamma r^{2}\right)= \\
=-2 \gamma r \rho(r, R)=-\frac{8 r}{\pi r^{2}} \rho(r, R)
\end{gathered}
$$

with boundary conditions:

$$
\begin{gathered}
V_{\text {nucl }}(0, R)=-4 /(\pi r), \\
y(0, R)=0 \\
\rho(0, R)=4 \gamma^{3 / 2} / \sqrt{\pi}=32 / R^{3}
\end{gathered}
$$

Further one can write the Dirac-Fock -like equations for a multi-electron system $\{$ core- $n l j\}$. Formally they fall into one-electron Dirac equations for the orbitals $n l j$ :

$$
\begin{aligned}
& \frac{\partial F}{\partial r}+(1+\chi) \frac{F}{r}-(\varepsilon+m-V) G=0 \\
& \frac{\partial G}{\partial r}+(1-\chi) \frac{G}{r}+(\varepsilon-m-V) F=0
\end{aligned}
$$

with large and small components $\mathrm{F}, \mathrm{G}$ and potential: $V(r)=2 V(r \mid$ core $)+V(r \mid n l j)+V_{e x}+V(r \mid R)$. and the Dirac quantum number:

$$
\chi=\left\{\begin{array}{c}
l, j=l-\frac{1}{2}, l^{\prime}=l-1 \\
-(l+1), j=l+\frac{1}{2}, l^{\prime}=l+1
\end{array} .\right.
$$

The potential $V(r)$ includes the electrical and polarization potentials of the nucleus. The part $V_{e x}$ accounts for exchange inter-electron interaction. The main exchange effect will be taken into account if in the equation for the $n l j$ orbital we assume $V(r)=V(r \mid l s)+V(r \mid n l j)$ and in the equation for the $n l j$ orbital $V(r)=V(r \mid$ core $)$. The rest of the exchange-correlation effects are accounted for in the first two PT orders by the total inter-electron interaction [6,7]. The core electron density is de- fined by iteration algorithm within gauge invariant QED procedure [10]. Let us consider further the calculating hyperfine structure constants. The constants are defined by the radial integrals (c.f.[7,15]):

$$
\begin{aligned}
A= & \left\{\left[(4,32587) 10^{-4} Z^{2} \mathrm{cg}_{I}\right] /\left(4 \mathrm{c}^{2}-1\right)\right\} \times \\
& \times \int_{0}^{\infty} d r r^{2} F(r) G(r) U\left(1 / r^{2}, R\right), \\
B= & \left\{7.287810^{-7} Z^{3} Q /\left[\left(4 \mathrm{c}^{2}-1\right) I(I-1)\right\} \times\right. \\
& \int_{0}^{\infty} d r r^{2}\left[F^{2}(r)+G^{2}(r) U\left(1 / r^{2}, R\right),\right.
\end{aligned}
$$

Here I is a spin of nucleus, $g_{I}$ is the Lande factor, $Q$ is a quadruple momentum of nucleus; radial integrals are calculated in the Coulomb units $(=3,57$ $10^{20} Z^{2} \mathrm{~m}^{-2} ;=6,17410^{30} Z^{3} \mathrm{~m}^{-3}$ ). Radial parts $F$ and $G$ of two components of the Dirac function for electron, which moves in the potential $V(r, R)+U(r, R)$, are defined by solution of the Dirac equations (perturbation theory zeroth order). The electric quadrupole spectroscopic hyperfine constant $B$ of an atomic state related to the electric field gradient $q$ and to the electric quadrupole moment $e Q$ of the nucleus in the following way: $B=e q Q / h$. So, in order to obtain the corresponding value of $Q$ it is necessary to combine the hyperfine structure constants data with the electric field gradient obtained from the QED perturbation theory formalism calculations in our approach.

We carried out the calculation of the hyperfine structure constants and nuclear quadrupole moment for radium ${ }_{88}^{223} R a$. In table 1 we present the calculated by us values of the magnetic dipole hyperfine structure constants $\mathrm{A}(\mathrm{MHz})$ of the $7 \mathrm{~s} 7 \mathrm{p}$ ${ }^{1} \mathrm{P}_{1},{ }^{3} \mathrm{P}_{1}$ and ${ }^{3} \mathrm{P}_{2}$ levels of ${ }_{88}^{223} R a$, compared with experiment, uncorrelated DF, MCDF (with account Breit and QED corrections), relativistic configuration interaction approach (with account correlations within random-phase approximation) RCI-RPA data [1].

Calculated values of magnetic dipole hyperfine structure constants $\mathrm{A}(\mathrm{MHz})$ of the $7 \mathrm{~s} 7 \mathrm{p}{ }^{1} \mathrm{P},{ }^{3} \mathrm{P}$ and ${ }^{3} \mathrm{P}$ levels of ${ }_{88}^{223} R a$, compared with experiment, uncorrelated DF, MCDF (with account Breit-

QED corrections), RCI-RPA data [1].

\begin{tabular}{|c|c|c|c|}
\hline Method & ${ }^{1} \mathrm{P}_{1}$ & ${ }^{3} \mathrm{P}_{1}$ & ${ }^{3} \mathrm{P}_{2}$ \\
\hline DF & $-226,59$ & 803,97 & 567,22 \\
\hline MCDF (Breit+QED) & $-330,3$ & 1251,9 & 737,1 \\
\hline RCI-RPA & $-242,4$ & - & - \\
\hline Present QED PT & $-339,1$ & 1209 & 704,5 \\
\hline Experiment & $-344,5(0,9)$ & $1201,1(0,6)$ & $699,6(3,3)$ \\
\hline
\end{tabular}


Let us note that the key quantitative factor of agreement between theory and experiment is connected with the correct accounting for the interelectron correlations, finite size niclear, Breit and QED radiative corrections [1-5,7,8,16,18].. The analysis shows that the interelectron correlation contribution to the hyperfine structure constant values is about $100-500 \mathrm{MHz}$ for different levels (see table 1). This fact explaines the low degree of accurace of the data, obtained within the standard uncorellated DF approach. At the same time the key difference between our approach and well known MCDF method is connected with the different degree of accounting for the interelectron correlations. The MCDF method is not gauge-invariant one and an accounting of multi-particle interelectron correlations is not fully fulfilled. This problem is in principle absent in our ap- proach (see the discussion regarding this topic in refs. $[10,14,21])$. From the other side, the contribution of the high order QED corrections and finite size nuclear effects can reach one-two dozens of $\mathrm{MHz}$ and more accurate account for these effects is preferable. Now the work on direct solving of the corresponding nuclear task with using shell model with the Woods-Saxon and spin-orbit potentials [20] is in progress. In table 2 we present the calculated and measured values of the nuclear electric quadrupole moment Q (barn) of ${ }_{88}^{223} R a$ by ISOLDE Collaboration in different ways, theoretically calculated values within the MCDF (with account Breit and QED corrections) method, relativistic many-body perturbation theory (RMBPT) approach and our QED PT formalism (from $[1,18]$ and refs there).

Table 2

The values of the nuclear electric quadrupole moment Q (barn) of ${ }_{88}^{223} R a$

\begin{tabular}{|c|c|}
\hline Method & Q (barn) \\
\hline MCDF (Breit+QED) & $1,21(0,03)$ \\
\hline ISOLDE Collaboration fs RaII & $1,254(0,003)\{0,066]$ \\
\hline Wendt et al, fs RaI & $1,19(0,12)$ \\
\hline RMBPT & 1,28 \\
\hline ISOLDE Collaboration fs RaI & $1,190(0,007)\{0,126\}$ \\
\hline ISOLDE Collaboration B(E2) & 1,2 \\
\hline Present QED PT & $1,22(0,03)$ \\
\hline
\end{tabular}

Our final result fits between the latest values obtained by the group of Wendt (ISOLDE Collaboration), but has a smaller error bar. So, we have carried out sensing and estimating the hyperfine structure parameters and nuclear quadrupole moment of the radium on the basis of gaugeinvariant QED PT with an account of the relativistic and correlation effects and reached sufficiently high accuracy.

Acknowledgement. Authors would like to thank very much Prof.A.Glushkov for proposing the work, support and information about the discussion with Prof. P.Pyykkö. The comments of anonymous reviewer are very appreciated.

\section{References}

1. Bieron J., Pyykkö P., Degree of accuracy in determining the nuclear electric quadrupole moment of radium//Phys.Rev. A. - 2005. - Vol.71. P.032502-1-8.

2. Bieron J., Pyykkö P., Jonsson P., Nuclear quadru- pole moment of ${ }^{201} \mathrm{Hg} / / \mathrm{Phys} . \mathrm{Rev}$. A. - 2005. Vol.71. - P.012502-1-8.

3. Schweppe J., Belkacem A., Blumenfeld L., Clayton N., Feinberg B., Gould H., Kostroum V.E., Levy L., Misawa S., Mowst J.R. and Priuor M.H. Measurement of the Lamb shift in lithiumlike uranium $\left(\mathrm{U}^{89+}\right) / /$ Phys.Rev.Lett. — 1998-Vol.66. — P.14341437.

4. Klaft I.,Borneis S., Engel T., Fricke B., Grieser R., Huber G., Kuhl T., Marx D., Neumann R., Schroder S., Seelig P., Volker L. Precision laser spectroscopy of ground state hyperfine splitting of H-like ${ }^{209} \mathrm{Bi}^{82+} / /$ Phys.Rev.Lett. - 1994. - Vol.73. P.2425-2427.

5. Ivanov L.N., Ivanova E.P., Kalinkin A.N. Nuclear and radiative effects in spectra of heavy and superheavy hydrogen-like ions//Nucl.Phys. - 1985. Vol.42. - P.355-362.

6. Ivanova E.P., Ivanov L.N., Glushkov A.V., Kramida A.E. High order corrections in the Relativistic Perturbation Theory with the model Zeroth Approximation, $\mathrm{Mg}$-like and $\mathrm{Ne}$-like ions // Phys.Scripta. - 1985. - Vol.32(4). - P.512-524.

7. A.V.Glushkov, S.V.Ambrosov, A.V.Loboda A.V., 
Yu.G.Chernyakova,

A.A.Svinarenko, O.Yu.Khetselius, QED calculation of the superheavy elements ions: energy levels, radiative corrections and hfs for different nuclear models// Nucl. Phys.A.: Nucl.and Hadr. Phys. -2004. — Vol.734. - P.21-24.

8. Khetselius O.Yu., Hyper fine structure of radium// Photoelectronics. - 2005. - N14. - P.83-85.

9. Glushkov A.V., Khetselius O., Consistent Quantum electrodynamics calcualtion of the fine and hyperfine structure parameters for heavy and super heavy multicharged Li-like ions// Preprint of I.I.Mechnikov Odessa National University. Institute of Physics: Ph-L-4. — Odessa-2003.

10. Glushkov A.V., Ivanov L.N. Radiation Decay of Atomic States: atomic residue and gauge non-invariant contributions // Phys. Lett.A. - 1992. Vol.170(1). - P.33-38.

11. Glushkov A.V., Rusov V.D., Ambrosov S.V., Loboda A.V., Resonance states of compound superheavy nucleus and EPPP in heavy nucleus collisions // New Projects and New Lines of research in Nuclear physics.Eds. Fazio G. and Hanappe F.,pp.142-154, Singapore, World Sci. (2003).

12. Glushkov A.V.,Malinovskaya S.V., Svinarenko A.A., Chernyakova Yu.G., QED Calculation of Electron Satellites Spectra in Intense Laser Field in Multicharged Ion//Int.J.Quant.Chem. — 2004. Vol.99(4). - P.936-939.

13. Glushkov A.V., Malinovskaya S.V., Co-operative laser nuclear processes: border lines effects// In: New projects and new lines of research in nuclear physics. Eds. G.Fazio and F.Hanappe, pp.242-260, Singapore : World Sci. (2003).

14. Glushkov A.V. Negative Ions of inert Gases// Pis'ma to JETP. — 1992. — Vol.55. — P.104-107; JETP Lett. — 1992. — Vol.55(2). — P.97-100.
15. Sobel'man I.I. Introduction to theory of atomic spectra. - Moscow: Nauka. - 1977.

16. Koshelev K.V., Labzowsky L.N., Tupitsyn I.I., The interelectron interaction corrections to the hyperfine structure of the 2p3/2 state in Li-like, B-like and N-like ${ }_{83}^{209} \mathrm{Bi}$ ions//J.Phys.B. - 2004. - Vol.37. - P.843-851.

17. Glushkov A.V., Gurnitskaya E.P., Loboda A.V., Advanced quantum mechanical calculation of superheavy ions: energy levels, Radiation and Finite Nuclear size effects// Low Energy Antiproton Phys., AIP Serie. — 2005. - Vol.796. - P.217220.

18. Glushkov A.V., Ambrosov S.V., Loboda A.V., Gurnitskaya E.P., Khetselius O.Yu., QED calculation of heavy multicharged ions with account for the correlation, radiative and nuclear effects// Recent Advances in Theor. Phys. and Chem. Systems. - 2006. - Vol.15. - P.285-300.

19. Glushkov A.V., Malinovskaya S.V., Gurnitskaya E.P., Khetselius O.Yu.,Dubrovskaya Yu.V., Consistent quantum theory of the recoil induced excitation and ionization in atoms during capture of neitron// J.Phys.CS. - 2006. - Vol.35. - P.425430.

20. Glushkov A.V., Malinovskaya S.V., Dubrovskaya Yu.V., Vitavetskaya L.A., Quantum calculation of cooperative muon-nuclear processes: discharge of metastable nuclei during negative muon capture// Recent Advances in Theor. Phys. and Chem. Systems. - 2006. - Vol.15. - P.301-324.

21. Glushkov A.V., Ambrosov S.V., Loboda A.V., Gurnitskaya E.P., Prepelitsa G.P., Consistent QED approach to calculation of electron-collision excitation cross-sections and strengths: Ne-like ions // Int. Journ.Quant.Chem. — 2005. — Vol.104, N4 . - P. 562-569. 